\title{
ALZHEIMER DISEASE
}

\section{Enhanced functional connectivity in AD after deep brain stimulation}

\begin{abstract}
Alzheimer disease $(\mathrm{AD})$ is one of the most common neurodegenerative disorders, causing a range of progressive neuropsychiatric symptoms and cognitive deficits. Researchers at the University of Toronto, Canada are currently investigating the potential of deep brain stimulation (DBS) for the treatment of $\mathrm{AD}$, and have now found evidence that DBS results in changes to neural circuitry and functional connectivity in patients with this condition.
\end{abstract}

\section{4 ...the brains ... showed increased glucose metabolism and functional connectivity... 77}

In a small phase I open-label trial, a research team led by Andres Lozano administered DBS of the fornix to five patients with mild, probable AD. In a follow-up study, the researchers used high-resolution PET to measure metabolic activity in different brain regions and performed a functional connectivity analysis. "These measures are very wellestablished as being sensitive to detecting changes in neural circuitry in the early stages of $\mathrm{AD}$, as well as detecting disease progression," explains Gwenn Smith, lead author of the follow-up study. Clinical outcomes were evaluated with the $\mathrm{AD}$ Assessment Scale-cognitive subscale
(ADAS-cog), a measure of cognition, memory and language ability, and on the Quality of Life-AD (QOL-AD) scale.

After 1 year of DBS, the brains of the patients showed increased glucose metabolism and functional connectivity compared with measurements taken at baseline. The changes in connectivity were localized in separate cortico-subcortical and cortico-hippocampal networks, and were accompanied by improvements in clinical and cognitive outcomes.

"The increases in metabolism were in contrast to the well-described metabolic decreases over 1 year in the course of $\mathrm{AD}$, and were more extensive than the effects of cholinesterase inhibitors over the same time interval," Smith points out. The authors note that declines in functional connectivity would also normally be expected over the longitudinal course of $\mathrm{AD}$.

These promising findings build on previous data from the same study that were published in 2010, and they highlight the potential for circuitry-based therapies in AD. The currently available medications for this condition are of limited efficacy, and clearance of amyloid- $\beta$ by antibodymediated therapy is not associated with clinical improvement.

"The persistent increases in metabolism after 1 year of DBS that are correlated with better clinical outcomes, as well as the increased functional connectivity,

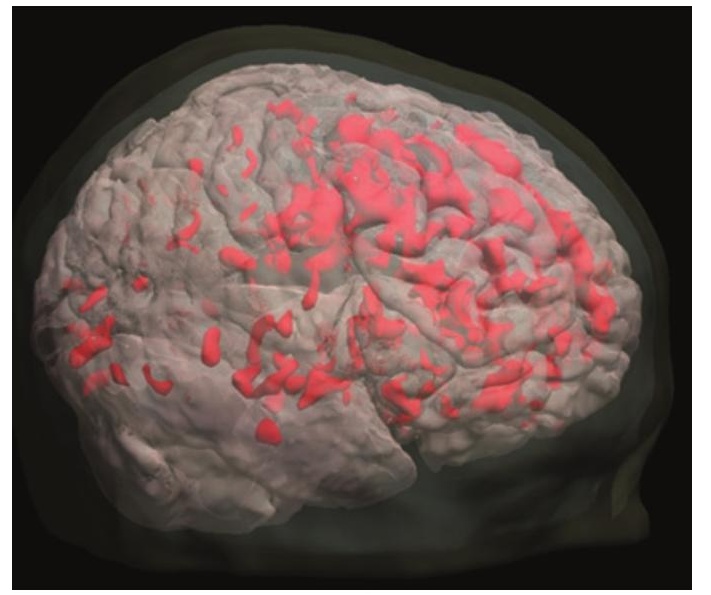

Increased glucose metabolism revealed by PET is correlated with improvements on ADAS-cog and QOL-AD scores. Image courtesy of G. S. Smith.

are encouraging," says Smith. “These preliminary results need to be replicated in a larger sample of patients in the early stages of AD." The researchers also plan to conduct basic research to investigate the mechanisms underlying the effects of DBS on memory circuits.

Ellen Bible

Original article Smith G. S.et al. Increased cerebral metabolism after 1 year of deep brain stimulation in Alzheimer disease. Arch. Neurol. doi:10.1001/ archneurol.2012.590

Further reading Laxton, A. W. et al. A phase I trial of deep brain stimulation of memory circuits in Alzheimer's disease Ann. Neurol. 68, 521-534 (2010) 